\title{
APPROXIMATION BY EQUIVARIANT HOMEOMORPHISMS. I
}

\author{
MARK STEINBERGER AND JAMES WEST
}

\begin{abstract}
Locally linear (= locally smoothable) actions of finite groups on finite dimensional manifolds are considered in which two incident components of fixed point sets of subgroups either coincide or one has codimension at least three in the other. For these actions, an equivariant $\alpha$-approximation theorem is proved using engulfing techniques. As corollaries are obtained equivariant "fibrations are bundles" and "controlled $h$-cobordism" theorems, as well as an equivariant version of Edwards' cell-like mapping theorem and the vanishing of the set of transfer-invariant $G$-homotopy topological structures, rel boundary, on $T^{n} \times D_{\rho}$ (when $T^{n}$ is the $n$-torus with trivial $G$ action and $D_{\rho}$ is a representation disc).
\end{abstract}

Here we consider locally linear (locally smooth [Br]) PL and topological actions of a finite group $G$ on $n$-manifolds. In addition, we require that a $G$-manifold, $M$, have gaps of codimension $\geq 3$ (i.e., for $H \subset G$, if $M_{\alpha}^{H}$ is a component of the $H$-fixed point set, $M^{H}$, and $M_{\alpha}^{>H} \subset M_{\alpha}^{H}$ is the subspace of points $x$ for which the isotropy subgroup, $G_{x}$, strictly contains $H$, then either $M_{\alpha}^{>H}=M_{\alpha}^{H}$ or $M_{\alpha}^{>H}$ has codimension $\geq 3$ in $M_{\alpha}^{H}$ ). We shall use the term "not necessarily locally linear $G$-manifold" when we wish to drop the local linearity hypothesis but retain the property that if $M_{\alpha}^{H} \subset M_{\beta}^{K}$ but $M_{\alpha}^{H} \neq M_{\beta}^{K}$ then $M_{\alpha}^{H}$ is a locally flat (inequivariantly) submanifold of $M_{\alpha}^{K}$ of codimension at least three.

This, while self-contained, is the second in a series of papers $\left[\mathbf{S W}_{\mathbf{2}, \ldots, \mathbf{8}}, \mathbf{S}\right]$ in which we analyze the extent of failure of the topological invariance of equivariant Whitehead torsion and the consequent failure of subgroups of the equivariant Whitehead group $\mathrm{Wh}_{G}^{P L}(M)$ of Illman $\left[\mathbf{I}_{\mathbf{1}}\right]$ (cf. Rothenberg $[\mathbf{R}]$ ) to classify PL or smooth $G$ - $h$-cobordisms up to topological equivalence (under our gap hypotheses the arguments of Browder and Quinn [BQ] and Rothenberg $[\mathbf{R}]$ show that $G-h$ cobordisms are classified up to PL or smooth equivalence by such subgroups). We eventually conclude in $\left[\mathbf{S W}_{\mathbf{7}}, \mathbf{S}\right]\left(\mathrm{cf}\right.$. $\left.\left[\mathbf{S W}_{\mathbf{2}}\right]\right)$ that the topological equivalence classes of $G$ - $h$-cobordisms on a $G$-manifold $M$ that are products over the union of fixed point components of $M$ having dimension $<5$ are classified by a group $\mathrm{Wh}_{G}^{\mathrm{Top}}(M)$ to which $\mathrm{Wh}_{G}^{P L}(M)$ maps homomorphically. The image in $\mathrm{Wh}_{G}^{\mathrm{Top}}(M)$ of the pertinent subgroup of $\mathrm{Wh}_{G}^{P L}(M)$ classifies up to equivariant homeomorphism, rel $M$, those $G$ - $h$-cobordisms on $M$ that admit equivariant handlebody decompositions relative to $M$. (This homomorphism is not generally surjective and there are $G-h$ cobordisms that do not admit such handle decompositions ( $\mathbf{c f}\left[\mathbf{S W}_{\mathbf{5}}, \mathbf{S}\right]$ ). In order

Received by the editors August 23, 1984 and, in revised form, July 7, 1986.

1980 Mathematics Subject Classification (1985 Revision). Primary 57S17, 57Q10, 55R05, 57R80; Secondary 57 N30.

Authors partially supported by NSF. 
to achieve our classification, we must develop a flexible tool for producing equivariant homeomorphisms which does not depend on explicit handle manipulations.

This is what we do in this paper: we develop the full equivariant analogue of the $\alpha$-approximation theorem of Chapman and Ferry [CF] and draw the expected corollaries, including an equivariant topological "thin $h$-cobordism theorem" with control in the base of the cobordism. Our results are equivariant in the strong sense that nonisovariant input data is used to produce $G$-homeomorphisms. Thus, our results do not follow automatically from the inequivariant ones by induction on strata. Moreover, many of the inequivariant arguments do not generalize directly, as relevant equivariant obstruction groups are nonzero. For instance, as shown in $\left[\mathbf{S W}_{\mathbf{2}}\right]$, even the topological Whitehead groups of the $n$-torus with the trivial action are nonzero (as are $\tilde{K}_{0_{G}}\left(S^{n}\right)$ for all $n$, and even $\tilde{K}_{0_{G}}^{\text {Top }}\left(S^{1}\right)$ ).

Our approach is to develop equivariant controlled engulfing techniques which enable us both to deduce an equivariant cell-like mapping theorem (Theorem 3 below) from the inequivariant theorem of Siebenmann [ $\left.\mathbf{S i}_{\mathbf{1}}\right]$ and to deduce the equivariant $\alpha$-approximation theorem from it by the Chapman "shuffle" as in $\left[\mathbf{C}_{\mathbf{1}}\right]$. In light of $[\mathbf{E}]$, in which Edwards gives a purely engulfing proof of the inequivariant cell-like mapping theorem, our results may be derived entirely through engulfing techniques.

We use "G-equivalence" to mean an equivariant homotopy equivalence. For an open $G$-cover, $\alpha$, of $Y$ (i.e., $g U \in \alpha$ for all $U \in \alpha$ ) we say that a $G$-equivalence $f: X \rightarrow Y$ is a $G$ - $\alpha$-equivalence provided $f$ is proper and there is an equivariant homotopy inverse for which the homotopies to the identity maps are limited by the covers $\alpha$ of $Y$ and $f^{-1} \alpha$ of $X$.

THEOREM 1 (EQUIVARIANT $\alpha$-APPROXIMATION). Let $N^{n}$ be a not necessarily locally linear $G$-manifold. Then for each open $G$-cover, $\alpha$, of $N$ there is an open $G$-cover, $\beta$, of $N$ such that every $G$ - $\beta$-equivalence, $f:\left(M^{n}, \partial M\right) \rightarrow(N, \partial N)$, from a (locally linear) $G$-manifold, $M$, of dimension $n$ that restricts to a homeomorphism, $f: M_{\gamma}^{N} \rightarrow N_{f(\gamma)}^{H}$, on all fixed point components (of $M$ or of $\partial M$ ) that have dimension $<5$ is equivariantly $\alpha$-homotopic to an equivariant homeomorphism.

ADDENDUM (EQUIVARIANT $\beta$-DOMINATION). By further restricting $\beta$, we need assume only that $f$ be a proper $G$ - $\beta$-domination (cf. $[\mathbf{F}]$ ), provided that $\operatorname{dim} M_{\gamma}^{H}=\operatorname{dim} N_{f(\gamma)}^{H}$ for each fixed-point component, $M_{\gamma}^{H}$, of $M$.

REMARK. The above theorem may be used to detect locally linear actions among the topological ones satisfying our standing dimension and codimension hypotheses.

Amusingly, we may now deduce a surgery-theoretic result similar to that used by Siebenmann in his proof of the cell-like mapping theorem. (See $[\mathbf{K S}$, Essay V, Appendix B] for a complete bibliography.)

COROLlaRY 1. Let $f: M \rightarrow T^{n} \times D_{\rho}$ be a G-homotopy topological structure, rel $\partial$, on the product of the $n$-torus with the trivial action and a representation disc with codimension 3 gaps (i.e., $M$ is a topological $G$-manifold and $f$ is a $G$ equivalence which restricts to a homeomorphism on the boundary). If $\operatorname{dim} D_{\rho}^{G}+n \geq$ 5 , then there is a covering, $p: T^{n} \rightarrow T^{n}$ such that the transfer

$$
(p \times 1)^{*} f:(p \times 1)^{*}(M) \rightarrow T^{n} \times D_{\rho}
$$

is G-homotopic rel $\partial$ to a $G$-homeomorphism. 
ProOF. Choose a cover $\beta$ of $T^{n} \times D_{\rho}$ such that any $G$ - $\beta$-equivalence is $G$ homotopic to a homeomorphism and such that near $T^{n} \times 0, \beta$ is of the form $\beta_{1} \times \varepsilon$ for some $\varepsilon$, with $\beta_{1}$ a cover of $T^{n}$. Now choose $p$ so that $(p \times 1)^{*}(M) \rightarrow T^{n} \times D_{\rho} \stackrel{\text { proj }}{\longrightarrow} T^{n}$ is a $G$ - $\beta_{1}$-equivalence, and radially deform $(p \times 1)^{*} f$ to be a homeomorphism over $T^{n} \times\left(D_{\rho}-(\varepsilon / 2) D_{\rho}\right)$.

We also get the expected equivariant analogue of the bundle theorem of [CF]. Here a $G$-fibration is a Hurewicz fibration in the category of $G$-spaces and a $G$ bundle is a bundle with fiberwise $G$-action which admits equivariant trivializations with the diagonal action over a cover by slices [LR]. We say that a $G$-space $X$ is equivariantly locally path connected if for each $x \in B$ and each slice neighborhood $U$ of $x$, there is a slice neighborhood $V \subset U$ of $x$ such that for each $y \in V$ there is a path in $U^{G_{y}}$ form $y$ to $x$.

THEOREM 2 (EQUIVARIANT BUNDLE THEOREM). Let $p: E \rightarrow B$ be a $G$ fibration where $E$ and $B$ are locally compact metric spaces and $B$ is locally finite dimensional and equivariantly locally path connected. Suppose that each $p^{-1} b$ is a compact $G_{b}$-manifold of dimension $n$ for which each fixed-point component has dimension $\geq 5$ and that the restriction of $p$ to $\bigcup_{b \in B} \partial p^{-1} b$ is a $G$-bundle. Then $p$ is a $G$-bundle.

We also obtain an equivariant version of Ferry's proof $[\mathbf{F}]$ of a conjecture of Kirby and Siebenmann. Here if $\beta$ is a $G$-cover of $X$, a $G$ - $\beta$-map $f: X \rightarrow Y$ is a $G$-map whose point inverses are limited by $\beta$.

COROLLARY 2. Let $\alpha$ be an open $G$-cover of the $G$-manifold $M^{n}$. Then there is an open $G$-cover $\beta$ of $M$ such that if $N^{n}$ is a $G$-manifold and $f:(M, \partial M) \rightarrow$ $(N, \partial N)$ is a proper $G-\beta$-map which restricts to a homeomorphism on strata of dimension less than 5 , then $f$ is $G$-homotopic to a $G$-homeomorphism through $\alpha$ maps.

Let $f: X \rightarrow Y$ be a $G$-map between $G$-ANR's $[\mathbf{J}, \mathbf{L}]$. We say that $f$ is $G$ cell-like (abbreviated $G$-CE) if for each $y \in Y$ and each $G_{y}$-neighborhood $U$ of $f^{-1} y$, the inclusion $f^{-1} y \subset U$ is $G_{y}$-nullhomotopic. (Equivalently, for each orbit $G / H \subset Y$ and each $G$-neighborhood $U$ of $f^{-1}(G / H)$, the inclusion $f^{-1}(G / H) \subset U$ $G$-deforms onto an orbit.) When $X$ and $Y$ are additionally separable and metric this is equivalent to $f$ being an equivariant fine homotopy equivalence (i.e., a $G-\alpha$ equivalence for all open $G$-covers $\alpha$ of $Y$ ) [SW $\mathbf{S}$ ], cf. [Hav].

We say that $f: X \rightarrow Y$ is an equivariant near homeomorphism if for each open $G$-cover $\alpha$ of $Y$ there is a $G$-homeomorphism $h: X \rightarrow Y$ which is $\alpha$-close to $f$. Our proof of Theorem 1 requires the following generalization of Siebenmann's cell-like mapping theorem $\left[\mathbf{S i}_{\mathbf{1}}\right]$.

Theorem 3 (Equivariant CELl-Like MapPing Theorem). Let $f$ : $(M, \partial M) \rightarrow(N, \partial N)$ be a $G-C E$ map (as pairs) from a $G$-manifold to a not necessarily locally linear $G$-manifold which restricts to a homeomorphism on all fixed point components $M_{\alpha}^{H}$ of dimension less than 5. Then $f$ is an equivariant near homeomorphism.

COMPLEMENT. For $f$ as above and $\alpha$ any open $G$-cover on $N$ there is an equivariant $\alpha$-homotopy $f_{t}: M \rightarrow N$ with $f_{1}=f$ and $f_{t}$ a $G$-homeomorphism for all $t<1$. 
In fact, we now get an equivariant version of Edwards CE mapping theorem [E] as an immediate corollary to $[\mathbf{E}]$ and Theorem 3 . For simplicity, we state only the "manifold without boundary" case.

COROLlARY (EQUIVARIANT EDWARDS THEOREM). Let $X$ be a locally compact $G-A N R$ such that for each unequal pair $X_{\alpha}^{H} \subset X_{\beta}^{K}$ of fixed point components, $X_{\alpha}^{H}$ has codimension $\geq 3$ and is $1-L C$ in $X_{\beta}^{K}$. Then a $G-C E$ map $f: M \rightarrow X$ of a $G$-manifold without boundary onto $X$ that embeds $M^{<5}$ may be approximated rel $M^{<5}$ by equivariant homeomorphisms if and only if each fixed point component $X_{\alpha}^{H}$ of dimension $\geq 5$ has the disjoint disks property.

(Here, $M^{<5}=\left\{M_{\alpha}^{H} \mid \operatorname{dim} M_{\alpha}^{H}<5\right\}$. A space $Y$ has the disjoint discs property if each pair of maps $f, g: D^{2} \rightarrow Y$ may be approximated by maps with disjoint images.)

The stratum by stratum induction in our argument leads us through an inequivariant extension of the $\alpha$-approximation theorem to locally flat manifold $k$-ads, which may be of independent interest. A locally flat manifold $k$-ad, $\left(N ; N_{1}, \ldots, N_{k}\right)$ in a manifold $N^{n}$ is a collection of submanifolds $N_{i} \subset N$ such that if $x \in \bigcap_{j=1}^{p} N_{i_{j}}$, there is a chart $\psi: \mathbf{R}^{n} \rightarrow N$ (or $\psi: \mathbf{R}_{+}^{n} \rightarrow N$ ) about $x=\psi(0)$ and a collection of linear subspaces, $V_{i_{j}} \subset \mathbf{R}^{n}$ such that $\psi \mid V_{i_{j}}$ (or $\psi \mid V_{i_{j}} \cap \mathbf{R}_{+}^{n}$ ) is a chart for $x$ in $N_{i_{j}}$ for $1 \leq j \leq p$. Thus, $\left(N ; N_{1}, \ldots, N_{k}, \partial N=N_{k+1}\right)$ is a locally flat $(k+1)$-ad, as is $\left(\partial N ; \partial N \cap N_{1}, \ldots, \partial N \cap N_{k}\right)$ a locally flat $k$-ad. We say that $\left(N ; N_{1}, \ldots, N_{k}\right)$ has codimension 3 gaps if each inclusion between components of intersections of $N_{i}$ 's (including $N=N_{0}$ ) is either the identity or has codimension at least three. Maps and homotopies of $k$-ads are required to preserve the $i$ th term for each $i$.

THEOREM 4 ( $\alpha$-APPROXIMATION FOR LOCALLY FLAT MANIFOLD $k$-ADS). Let $\left(N^{n} ; N_{1}, \ldots, N_{k}\right)$ be a locally flat manifold $k$-ad with codimension 3 gaps. Then for each open cover, $\alpha$, of $N$ there is an open cover, $\beta$, of $N$ such that each $\beta$ equivalence,

$$
f:\left(M^{n} ; M_{1}, \ldots, M_{k}, M_{k+1}=\partial M\right) \rightarrow\left(N ; N_{1}, \ldots, N_{k}, N_{k+1}=\partial N\right)
$$

of $(k+1)$-ads that restricts to a homeomorphism on all components of intersections of $M_{i}$ 's with corresponding components in $N$ of dimension less than 5 is $\alpha$-close to a homeomorphism, $h$, of $k$-ads. Moreover, if $f$ is a homeomorphism on $M_{i_{1}} \cap \cdots \cap M_{i_{p}}$, $h$ may be required to agree with it there.

REMARK. This is also true for locally flat $G$-manifold $k$-ads. Finally, our work in $\left[\mathbf{S W}_{\mathbf{2 , 4}, \mathbf{5}, \mathbf{7}}\right]$ requires the following controlled $h$-cobordism theorem. Here, $\left(W^{n+1}, M^{n}\right)$ is a $G$ - $h$-cobordism if $M$ and $W$ are $G$-manifolds with $M \subset \partial W$ a codimension 0 submanifold with equivariantly bicollared boundary such that $M \rightarrow W$ and $\overline{\partial W-M} \rightarrow W$ are proper $G$-equivalences. We say that $(W, M)$ is a $G-\alpha-h$ cobordism, for $\alpha$ a $G$-cover of $M$, if there is an equivariant $\alpha$-deformation retraction $r: W \rightarrow M$ such that the identity map of $\overline{\partial W-M}$ is equivariantly $(\beta, 1)$-connected over $M$ with control map $r$ (cf. $\left[\mathbf{Q}_{\mathbf{1}}\right]$ ). (That is, $r$ is a $G$-deformation retraction of $W$ onto $M$ whose tracks are limited by $r^{-1} \alpha$ and for each equivariant commutative 
diagram

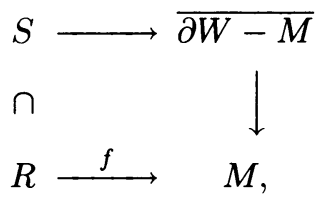

where $(R, S)$ is an equivariant relative 2-complex, there is an equivariant $\alpha$-lift of $f$ into $\overline{\partial W-M}$ rel $S$.) We say that $W$ is a $G$ - $\alpha$-product (with respect to $r$ ) if there is a $G$-homeomorphism $h:(M \times I, M) \rightarrow(W, M)$ whose tracks $m \times I$ are limited by $r^{-1} \alpha$ (cf. $\left.\left[\mathbf{Q}_{1}, \mathbf{C}_{2}\right]\right)$.

THEOREM 5 (EQUIVARIANT CONTROLLED $h$-COBORDISM THEOREM). Let $\alpha$ be an open $G$-cover of the $G$-manifold $M$. Then there is an open $G$-cover $\beta$ of $M$ such that if $(W, M)$ is a $G$ - $\beta$ - $h$-cobordism which is (coherently) a $G$ - $\beta$-product on all strata $($ of $W$ ) of dimension less than 6 , then $(W, M)$ is a $G$ - $\alpha$-product.

One of our main tools is an equivariant version of the deformation principle of Edwards and Kirby [EK], as generalized by Siebenmann [ $\left.\mathbf{S i}_{\mathbf{2}}\right]$. We discuss this and its corollaries in $\S 1$.

We give our controlled (isovariant) engulfing theorems in $\S 2$ and apply them to obtain a main engulfing lemma in $\S 3$. Theorems $3,1,2$, and 5 are proven in $\S \S 4,5$, 7 , and 8 , respectively, with Corollary 2 and the addendum to Theorem 1 given in $\S 6$.

We wish to thank Shmuel Weinberger for helpful conversations.

The results of this paper are true in greater generality. With adjustments of the hypotheses to fit the new categories (e.g., proper or isovariant homotopy conditions), they hold for (1) a certain class of locally universal $G$-actions on Hilbert cube manifolds $\left[\mathbf{S \mathbf { W } _ { \mathbf { 6 } }}\right]$ and (2) for proper Lie group actions on $n$-manifolds either with or without the codimensions hypothesis $\left[\mathbf{S} \mathbf{W}_{\mathbf{8}}\right]$.

1. Deformation of embeddings. In this section and the next, our $G$-manifolds need not satisfy gap hypotheses. All function spaces are assumed equipped with the compact-open topology. Given $G$-spaces $X$ and $Y$, we consider the conjugation action of $G$ on the space, $Y^{X}$, of all maps from $X$ to $Y$, i.e., $\left(g^{*} f\right)(x)=$ $g f g^{-1}(x)$, for $g \in G, f \in Y^{X}$, and $x \in X$. Thus, the fixed point set is the subspace of equivariant maps. Note that composition, $Z^{Y} \times Y^{X} \rightarrow Z^{X}$, is equivariant, and that the space, $H(X)$, of homeomorphisms of $X$ is a group in the category of $G$-spaces whenever composition and inversion are continuous.

We are concerned with equivariant deformations of $G$-subspaces of embeddings of (locally linear) $G$-manifolds. In this context, the work of Edwards and Kirby [EK] generalizes verbatim, as noted by Siebenmann [ $\left.\mathbf{S i}_{\mathbf{2}}\right]$. We find Siebenmann's setting of it in terms of stratified spaces convenient, but, not being concerned with actions of connected groups and not always assuming isovariant data, we eventually find it simplest for our purposes to work in the manifolds, rather than the orbit spaces. Thus, we review parts of $\left[\mathbf{S i}_{2}\right]$ in this context.

A $G$-stratified set is a metrizable $G$-space, $X$, equipped with a filtration by closed $G$-subspaces, $\varnothing=X^{(-1)} \subset X^{(0)} \subset \cdots \subset X^{(n)} \subset \cdots \subset X$, such that for each $n \geq 0$, the components of $X^{(n)}-X^{(n-1)}$ (which may be void) are open in $X^{(n)}$. The depth of the stratification is the length, if finite, of a maximal chain of components of pure strata, $X^{(n)}-X^{(n-1)}$, whose closures intersect. 
Such a space, $X$, is an equivariantly conelike stratified set $(G$-CS) provided that

(i) each component of $X^{(n)}-X^{(n-1)}$ is inequivariantly an $n$-manifold without boundary and has constant isotropy subgroup, and

(ii) each $x \in X^{(n)}-X^{(n-1)}$ has an open slice neighborhood in $X$ that is isomorphic as a $G_{x}$-stratified set to the product of a slice neighborhood of $x$ in $X^{(n)}-X^{(n-1)}$ with the open cone, $c L$, on a compact $G_{x}$-stratified set, $L$, of finite formal dimension (i.e., with a finite number of strata). More general definitions are obviously possible, but this one is convenient and provides for simple proofs.

EXAMPLES. (i) Let $M$ be a $G$-manifold, then $M$ is a $G$-CS set with $M^{(n)}-$ $M^{(n-1)}$ the union of all components of all $M^{H}-M^{>H}$ and $\partial M^{H}-(\partial M)^{>H}$ which have dimension $n$. Moreover, if $X$ is any union of $G$-translates of components of $M^{H}$ 's and $\left(M^{H} \cap \partial M\right)$ 's, then $X$ is a sub- $G$-CS set of $M$, as is $\partial M$. This is the orbit type stratification of $M$.

(ii) Let $N \subset M$ be a $G$-submanifold which is equivariantly locally flat in the sense that each point, $x$, in $N$ has a slice in $M$ that is $G_{x}$-homeomorphic to the product of a slice in $N$ with a $G_{x}$-representation. Then there is a $G$-CS structure on $M$ obtained by filtering both $N$ and $M-N$ as above. This generalizes in the obvious way to locally flat $G$-manifold $k$-ads.

(iii) Each locally compact $G$-simplicial complex is a $G$-CS set with the filtration given by its skeleta.

We now proceed with $\left[\mathbf{E K}, \mathbf{S i}_{\mathbf{2}}\right]$. For $C \subset U \subset X$, all $G$-spaces, let $\mathcal{E}(U, C ; X)$ be the space of open embeddings of $U$ in $X$ which restrict to the inclusion on $C$.

PROPOSITION 1.1. Let $U$ be an open $G$-neighborhood of a compact $G$-subspace, $C$, of a $G$-CS set, $X$. Let $A^{\prime}$ be a closed $G$-neighborhood of the closed $G$-subspace, $A$, of $X$. Then there is a $G$-neighborhood, $W$, of the inclusion, $i: U \rightarrow X$, in $\mathcal{E}\left(U, U \cap A^{\prime} ; X\right)$ and a $G$-deformation,

$$
h_{t}: W \rightarrow \mathcal{E}(U, U \cap A ; X),
$$

of $W$ into $\mathcal{E}(U, U \cap(A \cup C) ; X)$ such that

(i) for $f \in W$ and $x$ outside any preassigned compact $G$-neighborhood of $C$ in $U$, $h_{t}(f)(x)=f(x)$ for all $t$, and

(ii) for all sub-G-CS sets, $Y$, of $X$ and all $f \in W$ preserving or fixing $Y$, each $h_{t}(f)$ also preserves or fixes $Y$.

SKETCH OF PROOF. As in $\left[\mathbf{S i}_{\mathbf{2}}\right]$, it is enough to show that the proposition holds for $U$ an element of a $G$-basis for $X$, and the same pair of handle lemmas will suffice, with $X=G \times_{H}\left(R^{m} \times c L\right), U=G \times_{H}\left(10 B^{m} \times c_{10} L\right)$, and $H$ acting trivially on $R^{m}$. The remarks in the second paragraph of the section indicate that Siebenmann's argument for $X=H \times_{H}\left(R^{m} \times c L\right)$ is $H$-equivariant, and the rest follows by translation.

Recall that neighborhoods of a map, $f: X \rightarrow Y$, defined by majorant functions, $\varepsilon: Y \rightarrow(0, \infty)$, have a basis given by the subsets, $N_{\alpha}(f) \subset Y^{X}$, of maps $\alpha$-close to $f$ as $\alpha$ ranges over the open covers of $Y$. If $f$ is equivariant and $\alpha$ is a $G$-cover of $Y$, then $N_{\alpha}(f)$ is an invariant subset of $Y^{X}$.

COROllary 1.2. Let $X, U, A, A^{\prime}$, be as above with $i: U \subset X$, but let $C$ be closed and not necessarily compact. Let $\alpha$ be an open $G$-cover of $X$, and let $D$ be a closed neighborhood of $C$ in $U$. Then there are an open $G$-cover, $\beta$, of $X$ and 
$a$ a-deformation of $N_{\beta}(i) \cap \mathcal{E}\left(U, U \cap A^{\prime} ; X\right)$ into $\mathcal{E}(U, U \cap(A \cup C) ; X)$, with the deformation taking place in $N_{\alpha}(i) \cap \mathcal{E}(U, U \cap A ; X)$ and satisfying (ii) above as well as

(i') $h_{t}(f)(x)=f(x)$ for all $t$ and for all $x \in X-D$.

COROLLARY 1.3. The homeomorphism group, $\mathcal{H}(X)$, of a compact $G-C S$ set, $X$, is locally $G$-contractible. If $X$ is a noncompact $G$-CS set, then for each $G$-cover, $\alpha$, of $X$, there is a $G$-cover, $\beta$, of $X$ such that in $H(X), N_{\beta}(e)$ is null-homotopic in $N_{\alpha}(e), e$ being the identity.

The following is a simple generalization of [ $\mathbf{S i}_{\mathbf{2}}$, Theorem 6.5]. In it, $U$ and $X$ are $G$-CS sets, and $C \subset U$ is a closed, invariant set with compact frontier. $\mathcal{E}_{C}(U ; X)$ denotes the space of open embeddings, $f: U \rightarrow X$, for which $f(C)$ is closed, $\mathcal{E}(C ; X)$ is the space of closed embeddings of $C$ in $X$, and $p: \mathcal{E}_{C}(U ; X) \rightarrow$ $\mathcal{E}(C ; X)$ is restriction. If $B$ is a $G$-space, we say that it is equivariantly $(G-)$ path-connected if $B^{H}$ is path-connected for each subgroup, $H$, of $G$. Given an equivariant map, $j: B \rightarrow \mathcal{E}_{C}(U ; X)$, let $f_{b}=p j(b)$, and define $\pi_{b}: \mathcal{H}(X) \rightarrow \mathcal{E}(C ; X)$ by $\pi_{b}(h)=h f_{b}$.

COROLlARY 1.4 (ISOTOPY EXTENSION THEOREM). Let $B$ be an equivariantly path-connected $G$-space, and let $j: B \rightarrow \mathcal{E}_{C}(U ; X)$ be an equivariant map. If either $X$ has finitely many components or $B$ is $G$-locally connected, then the pullback $(p j)^{*} \pi_{b}$ is a G-locally trivial principal bundle with fiber $\mathcal{H}\left(X, \operatorname{rel} f_{b}(C)\right)$, for each $b \in B^{G}$. Moreover, the same conclusion holds for the subspaces of these mapping spaces comprised of those maps which preserve or fix any family of sub-G-CS sets of $X$.

Proof. Fix $\phi \in B^{G}$. For arbitrary $b \in B$, the argument in $\left[\mathbf{S i}_{\mathbf{2}}\right.$, Theorem 6.5 (I)] gives a $G_{b}$-equivariant section, $s_{b}$, of $(p j)^{*} \pi_{b}$, defined on a slice neighborhood, $N$, of $b$. Thus, for $x \in N, s_{b}(x) \in \mathcal{H}(X)$, and $s_{b}(x) f_{b}=f_{x}$. To get a local section of $\pi_{\phi}$ on $N$, we require a homeomorphism, $F_{b}: X \rightarrow X$, with $F_{b} f_{\phi}=f_{b}$. This will follow from the argument for the usual isotopy extension theorem [ $\mathbf{S i}_{\mathbf{2}}$, Theorem 6.5 (II)] applied to a $G_{b}$-equivariant path from $\phi$ to $b$.

COROLLARY 1.5 (ISOTOPY FACTORIZATION THEOREM). Let $\alpha$ be an open $G$-cover of the $G$-CS set $X$. Let $f: D_{\rho} \rightarrow \mathcal{H}(X)$ be equivariant and uniformly compactly supported, where $D_{\rho}$ is the unit disc of a linear representation $\mathbf{R}_{\rho}^{n}$ of $G$ over $\mathbf{R}$. Then there are $G$-maps $f_{1}, \ldots, f_{k}: D_{\rho} \rightarrow \mathcal{H}(X)$, with each $f_{i}$ supported on a compact subset of some $G U_{i}$ with $U_{i} \in \alpha$, such that $f(x) f(0)^{-1}=f_{1}(x) \circ \cdots \circ f_{k}(x)$ for all $x \in D_{\rho}$.

PROOF. Use an argument as given in [EK] for the zero dimensional representation and extend it by a Lebesgue number argument as in [ $\mathbf{S i}_{\mathbf{2}}$, Theorem 6.5 (II)], employing Corollaries 1.2-1.4 as needed.

REMARKS. In the above, we may replace $D_{\rho}$ by the universal $G$-Hilbert cube, $Q_{G}\left(\mathrm{cf} .\left[\mathbf{S W}_{\mathbf{6}}\right]\right)$, and hence by any compact $G$-AR.

As in $\left[\mathbf{S i}_{\mathbf{2}}\right.$, Theorem 6.17$]$ there is an equivariant extension of locally flat isotopies theorem. However, local flatness is difficult to verify unless the isotopy is supported on a stratum of the sub- $G$-CS set $Y \subset X$, in which case Corollary 1.5 yields a more finely controlled conclusion. 
COROLLARY 1.6. Let $Y$ be a sub-G-CS set of $X$ and let $f: D_{\rho} \rightarrow \mathcal{H}(Y)$ be an equivariant isotopy with support on a compact subspace $K$ of a pure stratum of $Y$, with $f_{0}=\mathrm{id}$. Then $f$ extends to an equivariant isotopy $F: D_{\rho} \rightarrow \mathcal{H}(X), F_{0}=\mathrm{id}$, with support on any preassigned compact neighborhood of $K$. Moreover, if $\varepsilon>0$ is given, we may assume that each $F\left(D_{\rho}\right)(x)$ is either constant or is contained in the $\varepsilon$-neighborhood of some $F\left(D_{\rho}\right)(y)$. Moreover, for each $\delta>0$ there is a neighborhood $U_{\delta}$ of $Y$ such that for each $x \in U_{\delta}$, there is a $y \in Y$ such that $F(-)(x)$ is $\delta$-close to $f(-)(g)$ as functions $D_{\rho} \rightarrow X$.

ProOF. Apply Corollary 1.5 to $f$ with respect to a small cover of $K$ by charts. Extend each of the resulting factors by an Alexander trick, damping them out along the cone coordinate on the cone on the link, $c L$, in the given chart, to be the constant isotopy outside of the $\gamma$-neighborhood of the cone point, where $\gamma$ depends on the number of factors.

COROLLARY 1.7. The same conclusion holds, with $\varepsilon$ and $\delta$ replaced by an open $G$-cover $\alpha$ and $\beta$ of $X$ and with $F$ supported on any closed neighborhood of $Y$, if $f$ is a finite composite of disjoint unions of isotopies supported on compact subsets of pure strata of $Y$.

2. Controlled equivariant engulfing. Once again, our $G$-manifolds need not satisfy gap hypotheses. Fix a $G$-manifold of dimension $n$ and a proper, equivariant control map, $p: M \rightarrow B$, where $B$ is a locally compact finite-dimensional metrizable space. In this section, we prove an isovariant engulfing theorem which combines the conclusions of $\left[\mathbf{I l}_{\mathbf{2}}, \mathbf{B i}\right.$, and $\left.\mathbf{S G H}\right]$.

Let $P$ be a $G$-PL space and let $f: P \rightarrow M$ be a proper, isovariant $G$-map. We say that $f$ is locally polyhedral if for each $x \in M$ there is a $G_{x}$-chart, $\alpha: \mathbf{R}_{\rho}^{n} \rightarrow M$, of $M$ around $x$ such that on $f^{-1}\left(\alpha\left(\mathbf{R}_{\rho}^{n}\right)\right)$, the composite of $f$ and $\alpha^{-1}$ is $G_{x}$-equivariantly PL.

The following trivially generalizes a lemma in [SGH].

LEMma 2.1. The image, $f(R)$, of a locally polyhedral map, $f: R \rightarrow M$, inherits unique equivariant $P L$ structure such that $f: R \rightarrow f(R)$ is $P L$ and $f(R) \subset$ $M$ is locally polyhedral.

Let $f: K \rightarrow M$ be a proper, isovariant map of a $G$-CW complex into $M$. We say $f$ has coherent codimension $\geq k$ if $\operatorname{dim} M_{f(\gamma)}^{H}-\operatorname{dim} K_{\gamma}^{H} \geq k$ for each component $K_{\gamma}^{H}$ of $K^{H}$.

Let $p \leq n-3$, and let $\mathcal{F}$ be a family of orbit types occurring in $M$. An engulfing structure, $(M, U)$, on $M$, of type $(\beta, p, \mathcal{F})$ consists of collections $U_{0} \subset \cdots \subset U_{p}$ and $M_{0} \subset \cdots \subset M_{p}$ of open subspaces of $M$, with $U_{i} \subset M_{i}$, such that if $f:(K, L) \rightarrow$ $\left(M_{i}, U_{i}\right)$ is a proper (in $\left.M\right)$ isovariant map of coherent codimension $\geq 3$, with $(K, L)$ a $G$-CW pair of relative dimension $\leq p-i$ and $K-L$ of type $\mathcal{F}$, then there is an isovariant $p^{-1} \beta$-homotopy, $f_{t}$, of $f, \operatorname{rel} L$ in $M_{i+1}$ to a map into $U_{i+1}$.

We first give a controlled version of the inequivariant engulfing theorem of [SGH], and then give some equivariant versions. (A full generalization will require an equivariant version of the controlled taming theory of Miller [Mil], Bryant [Bry], and Bryant and Seebeck [BS], and its applicability will depend on the existence of equivariant topological dual skeleta, due inequivariantly to Edwards (see [SGH]).) 
The PL requirement in the last sentence of the following is due to the lack of an $\varepsilon$-taming theory for half-spaces.

Proposition 2.2 (CONTROLled TOPOlOGiCAL ENGUlfing). Let $p \leq$ $n-3$ and let $\alpha$ be an open cover of the control space $B$. Then there is an open cover $\beta$ of $B$ such that for each enguifing structure $(M, U)$ on $M$ of type $(\beta, p)$ and each locally polyhedrally embedded pair $(P, Q)<\left(M_{0}, U_{0}\right)$ for which $\operatorname{dim} P \leq n-3$, $\operatorname{dim} P-Q \leq p, P \cap \partial M \subset Q$ and $\overline{P-Q} \subset M$ is a proper map, there is a $p^{-1} \alpha-$ isotopy, $h_{t}$, rel $Q$, of the identity map of $M$, supported on a closed (in $M$ ) subspace of $M_{p}$, with $P \subset h_{1}\left(U_{p}\right)$. When $M$ is $P L$ and $P \subset M$ is $P L$, the requirement that $P \cap \partial M \subset Q$ may be replaced by the requirements that $n>5, \operatorname{dim}(P \cap \partial M)<$ $n-3$, and that the homotopies $f_{t}$ given by the engulfing structure may be chosen to preserve the boundary.

PROOF. For $M$ topological, we may assume by passage to $(M-\partial M, U-\partial M)$ that $\partial M=\varnothing$. For simplicity, we may assume that $\alpha$ is relatively compact, so that any $p^{-1} \alpha$-homotopy of a proper map is proper. We argue by induction on $p$. For $p<n-3$, the result is almost immediate, using the global controlled taming theory of [SGH] to get the general position data to apply the proof of Bing's engulfing Theorem A [Bi] (details below). Here, $\beta$ may be any $p$-fold star refinement of $\alpha$. Briefly, let $R=\overline{P-Q}$, let $S=P \times 0 \cup R \times I$ and let $h: S \rightarrow M$, be induced by the homotopy $f_{t}$ of the engulfing structure. Let $H$ be a $p^{-1} \beta$-homotopy approximating $h$ rel $P \times 0$ which is locally polyhedral and in (Hudson-type $[\mathbf{H}]$ ) general position as in [SGH] and let $L$ be the union of the vertical line segments through points of the singular set of $H$. Since $\operatorname{dim} L<p$, induction gives an isotopy $k_{t}$ limited by the $(p-1)$-fold star, $\mathrm{St}^{p-1}\left(p^{-1} \beta\right)$, of $p^{-1} \beta$, with $L \subset k_{1}\left(U_{p}\right)$. Removing the interior of a regular neighborhood of $L$ in $\left(k_{1} H\right)^{-1}\left(U_{p}\right)$, we may assume $H$ is an embedding, and engulf up the vertical skeleta of a cylindrical (projection onto $P \times 0$ is simplicial) triangulation $T$ of $S$ whose simplices have small enough diameter that the resulting isotopy is limited by $p^{-1} \beta$.

For $p=n-3$, the proof is modeled on Bing's engulfing Theorem D [Bi]. Here, $\beta$ is a $(p+d+1)$-fold star refinement of $\alpha$, where $d=\operatorname{dim} B$, with the additional property that $\beta=\beta_{1} \cup \cdots \cup \beta_{d+1}$ where each $\beta_{i}$ is a discrete collection of subsets of $B$. Here, $\operatorname{dim} S=n-2$, so we may not be able to obtain a locally polyhedral approximation to $h$ as above. We do obtain a $p^{-1} \beta$-homotopy, $H$, approximating $h$, with the following properties with respect to a fine cylindrical triangulation $T$ of $S$ as above.

(1) $H$ is locally polyhedral and in general position on the $(n-3)$-skeleton, $T^{n-3}$, of $T$.

(2) For each $(n-2)$-simplex, $\sigma$, of $T$ there is a chart for which $H \mid \sigma \cup T^{n-3}$ is $\mathrm{PL}$, and $\sigma-\partial \sigma$ is in general position with respect to $T^{n-3}$.

(3) $H$ is simplicially nondegenerate on $T$ (i.e., $H$ embeds each simplex of $T$ ).

(4) There is a shadow $L \subset S$ for $H$, i.e., if $X_{1}$ is the closure of $\{x \in S \mid H(x)=$ $H(y)$ for some $\left.y \in T^{n-3}\right\}$ then $L$ is a $(p-1)$-dimensional union of vertical segments, containing $X_{1}$, such that if $x, y \in S$ with $H(x)=H(y)$, then $x \in L$ if and only if $y \in L$.

This data is sufficient for the engulfing. Induction gives a $\mathrm{St}^{p-1}\left(p^{-1} \beta\right)$ isotopy engulfing $\left(\left(P^{p-2} \times I\right) \cap S\right) \cup L$, and the argument above (modeled on Bing's engulfing 
Theorem B) gives a further $p^{-1} \beta$ isotopy engulfing $\left(P^{p-1} \times I\right) \cap S$. The (embedded) $(n-2)$-simplices of $T$ are now engulfed vertically in tracks along sets of $\beta_{1}, \beta_{2}$, etc., giving a $\mathrm{St}^{d}\left(p^{-1} \beta\right)$-isotopy engulfing $T^{n-3}$.

We first show how to obtain (1)-(3). Start with a locally finite cover $\mathcal{V}=\left\{V_{i}\right\}_{i \geq 0}$ of $M$ by charts. Choose a closure refinement of the cover $h^{-1} \mathcal{V}$ of $S$, and, via simplicial neighborhoods, choose a cover $C=\left\{C_{i}\right\}$ of $S$ by codimension zero PL subspaces with bicollared frontiers, $\partial C_{i}$ (which thus have dimension $\leq n-3$ ) such that $h\left(C_{i}\right) \subset V_{i}$ and $C_{i} \cap C_{j}=\partial C_{i} \cap \partial C_{j}$. Now choose a fine triangulation, $T_{1}$, of $S$, for which each $C_{i}$ is a subspace and choose an approximation, $h_{1}$, of $h$, satisfying (1), such that $h_{1}: C_{i} \rightarrow V_{i}$ is PL (after a possible change in the PL structure of $V_{i}$ ) and in general position. By Rushing's relative codimension $3 \varepsilon$-taming theorem $[\mathbf{R u}$, Theorem (5.5.2)] there is a small homeomorphism $g: V_{i} \rightarrow V_{i}, \operatorname{rel} C_{i} \cap T_{1}^{n-3}$, such that $g^{-1}\left(V_{i} \cap T_{1}^{n-3}\right)$ is PL. Using $g$ both to alter the PL structure of $V_{i}$ and to shift $h_{1}$ on the interior of the $(n-2)$-simplices of $C_{i}$, a small shift on the $(n-2)$-simplices, rel $\partial$, now achieves (1) and (2) for an approximation $h_{2}$. Now choose a cylindrical subdivision, $T$, of $T_{1}$ for which each $h_{2}: C_{i} \rightarrow V_{i}$ is simplicially nondegenerate (guaranteed by general position) and repeat the taming moves above inductively over the $(n-2)$-simplices of $T_{1}$ to regain (1) and (2) for the new triangulation, obtaining an approximation, $h_{3}$, satisfying (1)-(3).

Let $k=h_{3}$. By (1) and (2), $X_{1}$ is a subpolyhedron of $S$ of dimension $\leq n-5$, with $k \mid X_{1}$ PL onto $k\left(T^{n-3}\right)$. Let $L_{1}$ be the union of the vertical segments through $X_{1}$. Make another set of taming moves relative to $T^{n-3}$ so that each $(n-2)$ simplex is PL with respect to $L_{1}$ and shift each $(n-2)$-simplex $\sigma \operatorname{rel} k\left(T^{n-3}\right)$ so that $\sigma-\left(\partial \sigma \cup\left(\sigma \cap X_{1}\right)\right)$ is in general position with respect to $L_{1}$. Call the new map $k_{1}$ and note that we have not changed the intersections involving $T^{n-3}$ and that $k_{1}$ satisfies (1)-(3) with $T^{n-3}$ replaced by $T^{n-3} \cup L_{1}$ in (2). Let $X_{2}$ be the singularities of $k_{1}$ involving $T^{n-3} \cup L_{1}$ and let $L_{2}$ be the union of the vertical segments through $X_{2}$. Since $\operatorname{dim} L_{1} \leq n-4, \operatorname{dim}\left(X_{2}-X_{1}\right) \leq n-6$. Repeat the process inductively until $X_{j+1}=X_{j}$. The map $k_{j}$ will satisfy (1)-(4), with shadow $L_{j}$.

COROllary 2.3 (CONTROLled EQUiVARIANT ENGUlfing). Let $p \leq n-3$ and let $\alpha$ be an open $G$-cover of the control space $B$. Then there is an open $G$-cover, $\beta$, of $B$, such that for each engulfing structure $(M, U)$ on $M$ of type $(\beta, p, \mathcal{F})$, the following conditions on an equivariantly locally polyhedrally embedded pair $(P, Q) \subset$ $\left(M_{0}, U_{0}\right)$ provide for the existence of a $p^{-1}(\alpha)-G$-isotopy, $h_{t}, \operatorname{rel} Q$, of the identity map of $M$, supported on a closed (in $M$ ) subspace of $M_{p}$, for which $p \subset h_{1}\left(U_{p}\right)$.

(1) $P \subset M$ has coherent codimension $\geq 3, P-Q$ is of type $\mathcal{F}$ and of dimension $\leq p, \overline{P-Q} \subset M$ is a proper map, and for any stratum, $N$, of $M$ or $\partial M$ of dimension less than $5, P \cap N \subset Q$.

(2) If $M$ is topological or if $P \subset M$ is not $P L$, then each component of $P-Q$ has only one orbit type and $P \cap \partial M \subset Q$. If $M$ and $P \subset M$ are $P L$ and $P \cap \partial M \not \subset Q$ then $P \cap \partial M \subset \partial M$ satisfies (1), and the homotopies obtained from the engulfing structure may be chosen to respect the boundary.

PROOF. When $M$ and $P \subset M$ are PL, the general positioning for the argument above is given by Illman $\left[\mathbf{I}_{\mathbf{3}}\right]$. In the general case, we may consider each stratum of $P-Q$ disjointly from the others, and by passage to the strata of $(M, U)$, may 
assume that $P$ itself has a single orbit type and simply extend equivariantly the moves given by passage to the orbit space.

REMARKS. More general equivariant topological engulfing is at present useless, without the existence of equivariant topological dual skeleta. However, in case of need, we may obtain more general engulfing by a sequence of engulfing structures $\left(M^{1}, U^{1}\right) \subset \cdots \subset\left(M^{k}, U^{k}\right)$, where $k$ is the depth of the stratification (by dimension) of $M$ by orbit types, $\left(M^{i}, U^{i}\right)$ is an engulfing structure of type $\left(\beta, p_{i}, \mathcal{F}\right)$, where $\mathcal{F}$ is the family of fixed point components of a given dimension, and $\left(M_{p_{i}}^{i}, U_{p_{i}}^{i}\right) \subset\left(M_{0}^{i+1}, U_{0}^{i+1}\right)$.

3. The main lemma. Our main applications of controlled engulfing are given by the following lemma. Here, we consider $G$-ANR's $X$. (An action of a finite group $G$ on a finite-dimensional space $X$ is a $G$-ANR if $X$ and each $X^{H}$ are ANR's.) We denote by $X^{*}$ some closed, $G$-ANR subset of $X$ containing $\bigcup_{H \neq 1} X^{H}$ that is $1-L C$ embedded in $X$, i.e., for all $\varepsilon>0$ there is $\delta>0$ such that maps $f: S^{i} \rightarrow X-X^{*}$ of diameter $<\delta$ extend to maps of $D^{i+1}$ of diameter $<\varepsilon$.

LEMMA 3.1. Let $\alpha$ be an open $G$-cover of the $G$-ANR $N$ and let $V \subset N$ be a $G$-neighborhood of $N^{*}$. Then there is an open $G$-cover $\beta$ of $N$ and $a G$ neighborhood $V^{\prime} \subset V$ of $N^{*}$ such that if $M^{n}$ is a $G$-manifold and $f: M^{n} \rightarrow N^{n}$ is a G- $\beta$-equivalence which restricts to a homeomorphism $f: M^{*} \rightarrow N^{*}$, and $U$ is a $G$-neighborhood of $M^{*}$, then there is an equivariant $f^{-1} \alpha$-isotopy, $h_{t}$, of the identity map of $M$, supported on a closed (in $M$ ) subset of $f^{-1} V-M^{*}$, with $f^{-1}\left(\overline{V^{\prime}}\right) \subset h_{1}(U)$.

REMARKS. In the special case where $f$ is the identity map of $N$, the statement says precisely that the neighborhoods of $N^{*}$ in $N$ satisfy a controlled equivariant version of the $I$-axiom of [SGH]. Thus, $I$-regular neighborhoods may be used (with control) to study situations where Quinn's mapping cylinder neighborhood obstruction is nontrivial, which may be the case even for $G$-manifolds (e.g. $\left[\mathbf{Q}_{2}\right.$, 2.1.4]).

PROOF OF LEMMA 3.1. For simplicity of notation we shall assume unless stated otherwise that all inclusions of neighborhoods, $V \subset W$, have the property that $\bar{V} \subset W$.

We construct a sequence of neighborhoods of $N^{*}, V^{\prime}=V_{0, n-2} \subset \cdots V_{0,0} \subset$ $V_{1,0} \subset \cdots \subset V_{1,2}=V$ on which the size of $\beta$ will depend, such that for $f$ and $U$ as above,

(a) There is a sequence, $W_{0} \subset \cdots \subset W_{2}=U \cap f^{-1} V^{\prime}$ of neighborhoods of $M^{*}$ such that taking $M_{i}=f^{-1} V_{1, i}-M^{*}$ and $U_{i}=W_{i}-M^{*}, i=0,1,2$, gives an engulfing structure of type $(\eta, 2,\{e\})$, where the size of $\eta$ is determined by Corollary 2.3 and the argument below.

(b) Taking $M_{i}=f^{-1} V_{0,0}-M^{*}$ and $U_{i}=f^{-1}\left(V_{0,0}-\bar{V}_{0, i+1}\right)$ for $i=0, \ldots, n-3$ gives an engulfing structure of type $(\eta, n-3,\{e\})$.

To complete the proof, let $\varepsilon: M \rightarrow[0, \infty)$ be equivariant, with $\varepsilon^{-1}(0)=M^{*}$, so that, on $M=M^{*}$, the cover by $\varepsilon$-balls refines both $f^{-1} \beta$ and the distance from $M^{*}$. Choose equivariant topological dual skeleta (by pullback from the orbit space via [SGH]) $S^{2}$ and $T^{n-3}$ for $M-M^{*}$ with respect to $\varepsilon$.

Apply Corollary 2.3 via (a), with $P=$ the largest subcomplex of $S$ contained in $f^{-1}\left(V_{1,0}\right)$ and $Q=$ the largest subcomplex of $S$ contained in $W_{0}$, obtaining an 
isotopy $h_{t}$. Let $W_{-1} \subset W_{0}$ be a neighborhood of $M^{*}$ on which $h_{t}$ is the identity and let $W_{-2} \subset W_{-1}$ be such that the $\varepsilon$-neighborhood of $M-W_{-1}$ is contained in $M-W_{-2}$. The argument now proceeds as in [SGH, Theorem 2.1], with $Z=$ $f^{-1} \bar{V}_{0,0}-W_{-2}$ and $\hat{Z}$ equal to the closed $\varepsilon$-neighborhood of $Z$ : there is a small isotopy, $g_{t}$, of $1_{M}$, supported on a closed subspace of $f^{-1} V_{0,0}-M^{*}$, with $\hat{Z} \cap T \subset$ $g_{1}\left(M-f^{-1}\left(\overline{V^{\prime}}\right)\right)$. Successive applications of $h_{t}$, an $\varepsilon$-push, and $g_{t}^{-1}$ engulfs $f^{-1}\left(\overline{V^{\prime}}\right)$ by $W_{2}$.

We first construct $V_{1,0} \subset \cdots \subset V_{1,2}$. Choose $\beta_{1}$ small with respect to $\eta$ and choose neighborhoods $N_{0} \subset \cdots \subset N_{11}=V$ of $N^{*}$ such that each $N_{3 i+1}$ equivariantly $\beta_{1}$-deforms into $N^{*}$ within $N_{3 i+2}$ by a deformation $r_{i t}$ rel $N^{*}$. Such a sequence exists because $N$ and $N^{*}$ are $G$-ANR's. Let $\beta_{2}$ be any refinement of $\beta_{1}$ such that the $\beta_{2}$-neighborhood of each $\bar{N}_{i}$ is contained in $N_{i+1}$, and let $\beta$ be small enough that any $\beta$-equivalence $f$ as stated is a $\beta_{2}$-equivalence by maps and homotopies which extend the given homeomorphism $f \mid M^{*}$ and its inverse (i.e., $\beta$ is a star refinement of $\beta_{2}$ ). Write $g$ for the homotopy inverse just described. Set $V_{1,0}=N_{0}$ and $V_{1, i}=N_{3 i+3}$ for $i=1,2$.

Given $f$ and $U$ as above, and for any choice of $V^{\prime} \subset V_{1,0}$, choose neighborhoods $Z_{0} \subset \cdots \subset Z_{3}=U \cap f^{-1}\left(V^{\prime}\right)$ of $M^{*}$ such that for each $i<3$ there is an ambient $G$ - $f^{-1} \beta_{2}$-deformation, $D_{i t}$, of $M$ rel $M^{*}$, supported on $Z_{i+1}$, which carries $Z_{i}$ onto $M^{*}$ (again by ANR-ness). Let $h_{t}: 1_{M} \sim g f$ be a $\beta_{2}$-homotopy rel $M^{*}$. Then successive applications of $D_{i t}, h_{t}$ and $g r_{i t} f$ to a map $j:(K, L) \rightarrow\left(f^{-1} N_{3 i}, Z_{i}\right)$ provide a homotopy in $\left(f^{-1} N_{3(i+1)}, Z_{i+1}\right)$ to a map into $Z_{i+1}$, with the homotopy limited by $f^{-1}\left(\mathrm{St}^{4}\left(\beta_{1}\right)\right)$. Thus, there is a $f^{-1}\left(\mathrm{St}^{8}\left(\beta_{1}\right)\right)$-homotopy relative to $L$. Take $W_{0}=Z_{0}$ and $W_{i}=Z_{i+1}$ for $i=1,2$.

As $M^{*}$ is $1-L C$ in $M$, the second two stages satisfy the hypothesis for an engulfing structure, and we need only consider $\left.h:\left(K^{2}, L\right) \rightarrow\left(f^{-1}\right) V_{1,0}-M^{*}, W_{0}-M^{*}\right)$. Let $h: K \times I \rightarrow F^{-1} N_{3}$ be the $f^{-1}\left(\mathrm{St}^{8} \beta_{1}\right)$-homotopy given above, and triangulate $K \times I$ cylindrically so that each simplex is contained in a linear chart refining $f^{-1} \beta$. By the fine 1-equivalence we may assume that the 2-skeleton of $K \times I$ misses $M^{*}$, and by coning in the chart neighborhoods we may assume that each 3-cell, $\sigma$, meets $M^{\star}$ in at most one (interior) point $\dot{\sigma}$.

Let $\Sigma=\bigcup_{\sigma} \dot{\sigma}$ and for simplicity assume that $\Sigma \subset K \times I \stackrel{\text { proj }}{\longrightarrow} K$ is an embedding, and identify $\Sigma \times I$ with the union of the vertical segments from $\Sigma=\Sigma \times 0$ to $K \times 1$.

First adjust $h$ so that each $\dot{\sigma} \times I$ intersects $M^{*}$ only at $\sigma$ and $\Sigma \times(0,1]$ is locally polyhedrally embedded in $M-M^{*}$. Identifying $I$ with the face $v_{0} v_{1}$ of the standard 2-simplex $\Delta^{2}=\left(v_{0}, v_{1}, v_{2}\right)$ let

$$
h^{\prime}:\left(\Sigma \times \Delta^{2}, \Sigma \times v_{0} v_{2} \Sigma \times v_{1} v_{2}\right) \rightarrow\left(f^{-1} v_{1,0} ; M^{*}, W_{1}\right)
$$

be the extension of $h \mid \Sigma \times I$ obtained from the $f^{-1}\left(\mathrm{St}^{8} \beta_{1}\right)$-homotopy above, and adjusted rel $\Sigma \times\left(v_{0} v_{1} \cup v_{0} v_{2}\right)$ to embed $\Sigma \times\left(\Delta^{2}-v_{0} v_{2}\right)$ locally polyhedrally in $M-M^{*}$. Now approximate $h: K \times I-\Sigma \rightarrow f^{-1} V_{1,0}-M^{*}$ rel $\Sigma \times(0,1]$ so that $K \times I-\Sigma \times I$ is in general position with respect to $h^{\prime}\left(\Sigma \times\left(\Delta^{2}-v_{0} v_{2}\right)\right)$ and the approximation extends continuously via $h \mid \Sigma$.

By a move reminiscent of the "Alexander trick," we may slide the arcs $\dot{\sigma} \times I$ along the discs $\dot{\sigma} \times \Delta^{2}$.rel $\dot{\sigma}$, obtaining a new $h$ whose intersection with $M^{*}$ is precisely $\Sigma \times I$. Precomposing $h$ with a slide in the domain which pulls $K \times I$ off a small regular neighborhood of $\Sigma \times I$, we obtain a homotopy as desired which misses $M^{*}$. 
The choice of $V_{0, n-2} \subset \cdots \subset V_{0,0}$ is similar to Quinn's construction of an equivariant $(\eta, n-2, \varnothing)$ tame structure of the $N-N^{*}$ over $N^{*}\left[\mathbf{Q}_{\mathbf{1}}\right]$. (Quinn observes in $\left[\mathbf{Q}_{\mathbf{2}}, 2.2 .1\right]$ that such a structure exists. We simply modify his proof for our purposes, and refer the reader to $\left[\mathbf{Q}_{\mathbf{1}}\right]$ for the necessary definitions and ingredients.) First choose $V_{0,0} \subset V_{1,0}$, a retraction $e: V_{0,0} \rightarrow N^{*}$, and an open $G$-cover $\eta^{\prime}$ of $N^{*}$ so that $e^{-1} \eta^{\prime}$ refines $\eta$. By the locally compact version of Quinn's eventual Hurewicz theorem $\left[\mathbf{Q}_{\mathbf{1}}, 5.2\right]$, there is an open $G$-cover, $\mu$, of $N^{*}$, such that the composite of a suitable sequence of "relatively homologically $(\mu, n-3)$ trivial" maps over $N^{*}$ is "relatively $\left(\eta^{\prime}, n-3\right)$ connected." Notice that since Quinn's proof of the eventual Hurewicz theorem proceeds skeleton by skeleton it applies verbatim to equivariant maps of free $G$-pairs over $N^{*}$. We only give the construction of $V_{0,2} \subset V_{0,1} \subset V_{0,0}$, as the others are built analogously.

As in $\left[\mathbf{Q}_{\mathbf{1}}, 5.3\right]$ it suffices to construct

$$
V_{0,0}=V_{0,0,-2} \supset V_{0,1,-1} \supset V_{0,1,0}=V_{0,1} \supset V_{0,1,1} \supset \cdots \supset V_{0,1,2^{k+2}}=V_{0,2},
$$

where $k=n-3$, such that

(1) each $f^{-1}\left(\bar{V}_{0,1, i}-\bar{V}_{0,1, i+1}\right) \subset f^{-1}\left(\bar{V}_{0,1, i-1}-\bar{V}_{0,1, i+2}\right)$ is $(\mu, 1)$-connected over $N^{*}$, and

(2) each $f^{-1}\left(\bar{V}_{0,1, i+1}\right)$ is $(e f)^{-1} \mu$-deformable into $M^{*}$ rel $M^{*}$, with the deformation taking place in $f^{-1}\left(V_{0,1, i}\right)$ (giving rise to a controlled homological tame structure by the argument of $\left.\left[\mathbf{Q}_{\mathbf{1}}, 3.1 .1\right]\right)$.

Quinn shows how to do this $\left[\mathbf{Q}_{\mathbf{1}}, 5.4\right]$ when $f$ is the identity map. We use Quinn's argument in $N$ with a suitable refinement, $\mu^{\prime}$, of $\mu$, together with buffer neighborhoods and $\beta$-homotopy data as in the argumentation for case (a) above to deduce that (1) and (2) hold in $M$.

4. Proof of the equivariant CE-mapping theorem. We first note that both here and in the proof of the equivariant $\alpha$-approximation theorem we may assume, by passage to the double and Corollary 1.2, that our manifolds are without boundary. We make this assumption both here and in $\S 5$.

As shown in $\left[\mathbf{S i}_{\mathbf{1}}\right]$, the complement follows from the main statement by Corollary 1.3 , so it suffices to show approximability by homeomorphisrns. We argue stratum by stratum, noting that since a $G$-CE map $f: M \rightarrow N$ restricts on fixed-point components $f_{\gamma}^{H}: M_{\gamma}^{H} \rightarrow N_{\gamma}^{H}$ to a $W_{\gamma}$-CE map (where $W_{\gamma}$ is the isotropy subgroup of $\gamma \in \pi_{0} M^{H}$ under the action of $\left.W(H)=N(H) / H\right)$, the dimensions of $M_{\gamma}^{H}$ and $N_{\gamma}^{H}$ are equal by the Vietoris-Begle theorem [Sp].

Our argument uses the inequivariant CE mapping theorem [Si $\mathbf{S i}_{\mathbf{1}}$ ], Lemma 3.1, and the equivariant Bing shrinking criterion. The statement and proof of the latter is a trivial generalization of the inequivariant version in Chapman $\left[\mathbf{C}_{\mathbf{3}}\right.$, Theorem 26.1].

Lemma 4.1 (Equivariant Bing Shrinking Criterion). Let $f: X \rightarrow Y$ be a proper surjective $G$-map between locally compact $G$-spaces. Then $f$ is an equivariant near homeomorphism if and only if for each open $G$-cover $U$ of $X$ and each open $G$-cover $\mathcal{V}$ of $Y$ there is an equivariant homeomorphism $h: X \rightarrow X$ such that $f h$ is $\mathcal{V}$ close to $f$ and each $h\left(f^{-1}(y)\right)$ lies in some element of $\mathcal{U}$. 
Thus, assume, after taking a suitable approximation, that $f$ is a homeomorphism on all strata smaller that $M_{\gamma}^{H}$. We shall approximate $f$ by a map which is a homeomorphism $M_{\gamma}^{H} \rightarrow N_{\gamma}^{H}$.

Let $D$ be the decomposition of $M$ whose only nontrivial elements are the sets $M_{\gamma}^{H} \cap f^{-1} y$ for $y \in N_{\gamma}^{>H}$ and their $G$-translates. Then $f$ factors through a $G$-CE map $\bar{f}: M / D \rightarrow N$. We shall show that the projection map $\pi: M \rightarrow M / D$ is a near homeomorphism, so that we may assume in the subsequent argument that $f^{-1} N_{\gamma}^{>H} \cap M_{\gamma}^{H}=M_{\gamma}^{>H}$.

Let $U$ and $\mathcal{V}$ be covers of $M$ and $M / D$ as in the hypothesis of Lemma 4.1. Let $\mathcal{V}^{\prime}$ be an equivariant collection of open sets in $N$ which covers $G N_{\gamma}^{>H}$, such that $\bar{f}^{-1} \mathcal{V}^{\prime}$ refines $\mathcal{V}$ and let $V$ be a $G$-neighborhood of $G N_{\gamma}^{>H}$ in $N$ whose closure is covered by $\mathcal{V}^{\prime}$.

Let $U$ be a $G$-neighborhood of $G M_{\gamma}^{>H}$ in $M$ such that $U \cap f^{-1} \mathcal{V}^{\prime}$ refines $U$. The main lemma provides a $W_{\gamma}$-equivariant $f^{-1} \mathcal{V}^{\prime}$-isotopy, $h_{t}$, of $M_{\gamma}^{H}$ rel $M_{\gamma}^{>H}$, supported on $f^{-1} V$, such that each set in $D \cap M_{\gamma}^{H}$ is contained in $h_{1}\left(U \cap M_{\gamma}^{H}\right)$. As $h_{t}$ is constructed from small pushes in $M_{\gamma}^{H}-M_{\gamma}^{>H}$, Corollary 1.7 provides an extension of $h$ to an equivariant $f^{-1} \mathcal{V}^{\prime}$-isotopy of $1_{M}$ supported on $f^{-1} V$ (hence a $\pi^{-1} \mathcal{V}$-isotopy) $\bar{h}_{t}$. The required shrinking is given by $\bar{h}_{1}^{-1}$.

Thus, we shall assume that $f^{-1} N_{\gamma}^{>H} \cap M_{\gamma}^{H}=M_{\gamma}^{>H}$. Let $D^{\prime}$ be the decomposition of $M$ whose only nontrivial elements are $M_{\gamma}^{H} \cap f^{-1} y$ for $y \in N_{\gamma}^{H}-N_{\gamma}^{>H}$ and their $G$-translates. As above, is suffices to show that $\pi: M \rightarrow M / D_{1}$ is a near homeomorphism.

Given $\mathcal{U}$ and $\mathcal{V}$ as above, choose refinements $\mathcal{U}^{\prime}$ and $\mathcal{V}^{\prime}$ of $\mathcal{U} \cap f^{-1}\left(N-G N_{\gamma}^{>H}\right)$ and $\mathcal{V} \cap \bar{f}^{-1}\left(N-G N_{\gamma}^{>H}\right)$, respectively, with $\mathcal{V}^{\prime}$ sufficiently fine that any self-map of $M-f^{-1} G N_{\gamma}^{>H}$ which is $\pi^{-1} \mathcal{V}^{\prime}$-close to the identity map extends continuously by the identity map of $f^{-1} G N_{\gamma}^{>H}$.

Consider $\pi: M_{\gamma}^{H}-M_{\gamma}^{>H} \rightarrow\left(M_{\gamma}^{H}-M_{\gamma}^{>H}\right) / D \cong N_{\gamma}^{H}-N_{\gamma}^{>H}$. Here, $W_{\gamma}$ acts freely and the induced map of orbit spaces is CE. Lifting the homotopy obtained from the inequivariant complement of the CE mapping theorem, we obtain a $W_{\gamma}$-equivariant homotopy $f_{t}$, with $f_{1}=f$ and $f_{t}$ a homeomorphism for $0 \leq t<1$, with the homotopy limited by $f^{-1} \mathcal{V}^{\prime \prime}$, for $\mathcal{V}^{\prime \prime}$ a star refinement of $\mathcal{V}^{\prime}$. Then for any $t_{0}<1$, $f_{0}^{-1} f_{t}$ is a $\pi^{-1} \mathcal{V}^{\prime}$-isotopy from the identity map to $f_{0}^{-1} f_{t_{0}}$. For $t_{0}$ sufficiently close to $1, f_{0}^{-1} f_{t_{0}}$ shrinks sets in $D_{1}$ into sets in $U^{\prime}$. Moreover, the construction of $f_{0}^{-1} f_{t}$ given by $\left[\mathbf{S i}_{\mathbf{1}}\right]$ is as a composite of compactly supported moves, so an equivariant extension $g: M \rightarrow M$ of $f_{0}^{-1} f_{t_{0}}$ exists by Corollary 1.7 , and shrinks $D_{1}$ as required.

5. Proof of the equivariant $\alpha$-approximation theorem. As above, we may assume that our manifolds are without boundary, and the following lemma gives the dimension requirements for a stratum by stratum induction.

LEMMA 5.1. Let $\alpha$ be an open $G$-cover of the $G$-manifold $N^{n}$. Then there is an open $G$-cover $\beta$ of $N$ such that if $f: M^{n} \rightarrow N^{n}$ is a $G$ - $\beta$-equivalence which restricts to a homeomorphism on all strata of dimension less than 5 , then the restriction to each fixed-point component $f_{\gamma}^{H}: M_{\gamma}^{H} \rightarrow N_{\gamma}^{H}$ is $\alpha$-close to an inequivariant homeomorphism.

ProOF. Since $f_{\gamma}^{H}$ is a $W_{\gamma}$ - $\beta$-equivalence, $\left[\mathbf{C}_{\mathbf{1}}\right.$, Theorem 1$]$ provides that for $\beta$ sufficiently small, $f_{\gamma}^{H}$ is close to an inequivariant approximate fibration. Since the 
homotopy fiber of $f_{\gamma}^{H}$ is trivial, this approximate fibration is a cell-like map. The result follows from $\left[\mathbf{S i}_{\mathbf{1}}\right]$ and the Vietoris-Begle theorem $[\mathbf{S p}]$.

In fact, this sketch is a paradigm for our argument. We show that if $f_{\gamma}^{>H}$ is already a homeomorphism, $\alpha$ is given, and $\beta$ is sufficiently small, then $f_{\gamma}^{H}$ is $\alpha$-close to a $W_{\gamma}$-CE map. Our constructions extend equivariantly over $M$ with control, and the induction is completed by the (inductive) proof of the $G$-CE mapping theorem.

For simplicity, we replace $M_{\gamma}^{H}, N_{\gamma}^{H}, M_{\gamma}^{>H}, N_{\gamma}^{>H}$ and $W_{\gamma}$ by $M, N, M^{*}, N^{*}$ and $G$, respectively.

As mentioned in the introduction, we shall adapt Chapman's argument $\left[\mathbf{C}_{\mathbf{1}}\right]$ to show that for any $\alpha$ there is a $\beta$ such that if $f: M \rightarrow N$ is a $G-\beta-1$ equivalence, with $f^{*}: M^{*} \rightarrow N^{*}$ a homeomorphism, then for any open $G$-cover $\gamma$ of $N$ there is a $G$ - $\gamma$-equivalence $f^{\prime}: M \rightarrow N$ which is $\alpha$-close to $f$. The required $G$-CE map is then obtained as a limit of a convergent sequence of such $f^{\prime}$.

REMARKS. Our engulfing techniques permit a full equivariant analogue of Chapman's Theorem 1 (approximation by approximate fibrations) if $M$ has "stable" gaps (i.e., $2 \operatorname{dim} M_{\gamma}^{>H}<\operatorname{dim} M_{\gamma}^{H}$ whenever $M_{\gamma}^{>H} \neq M_{\gamma}^{H}$ ), by using topological dual skeleta of dimension less than the gap codimension and general position.

Our adaptation of Chapman's handle lemma $\left[\mathbf{C}_{\mathbf{1}}\right.$, Theorem 5.2] is the following. Here, generalized singular set corresponds to $M^{*}, N^{*}$, etc.

LEMMA 5.2. For each $\varepsilon>0$ and $n \geq 0$ there is a $\delta>0$ such that for each $\mu>0$ there exists $\nu>0$ such that if $M^{n}$ is a $G$-manifold and $f: M \rightarrow \mathbf{R}^{n-k} \times \mathbf{R}_{\rho}^{k}$ is a homeomorphism on generalized singular sets, an equivariant $\delta$-equivalence over $B_{3}^{n-k} \times D_{3}^{k}$ and an equivariant $\nu$ equivalence over $B_{3}^{n-k} \times\left(D_{3}^{k}-\stackrel{\circ}{D}_{1 / 3}^{k}\right)$, then there is a map $\tilde{f}: M \rightarrow \mathbf{R}^{n-k} \times \mathbf{R}_{\rho}^{k}$ which is an equivariant $\mu$-fibration over $B_{1}^{n-k} \times D_{1}^{k}$ and is equivariantly $\varepsilon$-homotopic to $f \operatorname{rel} M-f^{-1}\left(\stackrel{\circ}{B}_{3}^{n-k} \times \stackrel{\circ}{D}_{2 / 3}^{k}\right)$.

Here $B_{t}^{n-k}$ is the unit ball of radius $t$ in $\mathbf{R}^{n-k}$ and $D_{t}^{k}$ is the unit ball of radius $t$ in $\mathbf{R}_{\rho}^{k}$.

The argument now follows by a standard induction down the dual handle structure in each element of a cover of $N$ by charts. For a cell $\sigma^{n-k}$ of type $H$, we apply the handle lemma $H$-equivariantly to $\mathbf{R}^{n-k} \times \mathbf{R}_{\rho}^{k}$, where $\mathbf{R}_{\rho}^{k}$ is the open dual cell of $\sigma$ and $\mathbf{R}^{n-k}$ lies in the interior of $\sigma$, and obtain $G$-equivariance by translation. Thus, the induction starts with the action of the trivial group on $\mathbf{R}^{n}$. Here, there is no statement about $\nu$, but a direct application of Chapman's Lemma 5.1 produces a map which is a $\mu$-fibration over $B_{1}^{n}$. In fact, by our codimension 3 gap hypothesis, equivariance does not enter until $k=3$. At each stage, $\mu$ is the $\nu$ produced by the previous stage and the $\beta$-control has been relaxed by a factor of $2 \varepsilon$. For a given $\varepsilon$ and small enough $\beta$, we get a small deformation to a $\mu$-fibration for any $\mu$ as required.

The handle lemma is proven by Chapman's shuffle argument, in which the problem is first localized over an embedded $T^{n-k-1} \times \mathbf{R} \times \mathbf{R}_{\rho}^{k} \subset \mathbf{R}^{n-k} \times \mathbf{R}_{\rho}^{k}$, wrapped up over $T^{n-k} \times \mathbf{R}_{\rho}^{k}$, and unfurled over $\mathbf{R}^{n-k} \times \mathbf{R}_{\rho}^{k}$. Chapman uses the unfurled map and a radial contraction of the base to get improved control over part of the target, and controlled engulfing to cover a translation shifting the product of unit balls into the region of improved control. 
The controlled engulfing for both this move and the wrapping up is accomplished by the following lemma. Here we are given $D={\stackrel{\circ}{D_{\rho}^{n-1}}}^{n}$, and equivariant map $\phi: D \rightarrow[0, \infty)$, and an isotopy $\theta_{t}: \mathbf{R} \rightarrow \mathbf{R}, t \geq 0$, supported on $[-1,1]$, with $\theta_{t}=\mathrm{id}$ for $t \geq 1$. Let $\theta: D \times \mathbf{R} \rightarrow D \times \mathbf{R}$ be given by $\theta(x, y)=\left(x, \theta_{\phi(x)}(y)\right)$, let $D_{t}=\phi^{-1}([0, t])$ and let $\pi: D \times \mathbf{R} \rightarrow D$ by the projection.

LEMMA 5.3. For every $\varepsilon>0$ there is a $\delta>0$ such that if $f: M^{n} \rightarrow D \times \mathbf{R}$ is an equivariant map which is a $G-\delta$-equivalence over $D_{4} \times[-4,4]$ and a homeomorphism on generalized singular sets, then there is an equivariant $(\pi f)^{-1} \varepsilon$-isotopy $\tilde{\theta}_{t}$ of the identity map of $M$, supported on $f^{-1}\left(D_{2} \times[-2,2]\right)$ such that $f \tilde{\theta}_{1}$ is $\varepsilon$-close to $\theta f$. Additionally, if $\mu>0$ there is $a \nu>0$ such that if $f$ is a $\nu$-equivalence over $\left(D_{4}-\stackrel{\circ}{D}_{1 / 3}\right) \times[-4,4]$, then we may require that $f \tilde{\theta}_{1}$ is $\mu$-close to $\theta f$ over $\left(D_{4}-\stackrel{\circ}{D}_{2 / 3}\right) \times \mathbf{R}$.

ProOF. In $\left[\mathbf{C}_{\mathbf{1}}\right.$, Theorem 3.6] Chapman works his analogue of this up from an easier lemma by the method of partitioning $[-1,1]$ as $-1=x_{0}<\cdots<x_{k}=1$ and considering the laminae $\theta\left(D \times\left[x_{i-1}, x_{i}\right]\right)$ and the tubes $\pi^{-1} \beta$ for a sufficiently fine open cover $\beta$ of $D$. He obtains his homeomorphism $\tilde{\theta}_{1}$ by using engulfing to move each $f^{-1}\left(D \times x_{i}\right)$ into $f^{-1} \theta\left(D \times\left(x_{i-1}, x_{i}\right)\right)$ with movement guided by the tubes $(\pi f)^{-1} \beta$. The result is, for sufficiently fine partitions and sufficiently fine $\beta$, sufficiently well controlled. (This is because the control is mediated by the thickness of the laminae and by $\mathrm{St}^{p} \beta$, where $p=p(n, k)$ comes from repeated engulfing control estimates. Thus, given $\mu$, we can choose a sufficiently fine partition, and choose $\beta$ to be sufficiently fine near $D-\stackrel{\circ}{D}_{2 / 3}$, that the laminar chambers of $\pi^{-1}\left(\mathrm{St}^{p} \beta\right)$ in $\left(D-\stackrel{\circ}{D}_{2 / 3}\right) \times \mathbf{R}$ have diameter less than $\frac{1}{3} \mu$. If we now choose $\nu$ so small that each subset, $A$, of $\left(D-\stackrel{\circ}{D}_{2 / 3}\right) \times[-1,1]$ of diameter $<\nu$ is contained in $\beta_{i} \times\left[x_{i-1}, x_{i}\right]$ for some $\beta_{i} \in \beta$, then the diameter of $\theta(A)<\mu$ and the $\nu$-equivalence stays a $\mu$-equivalence.)

All of the above considerations go over verbatim to the equivariant context once we establish the equivariant analogue of Chapman's simpler lemma $\left[\mathbf{C}_{\mathbf{1}}\right.$, Lemma 3.4 ], which is as follows, using the notation of Lemma 5.3 above.

LEMMA 5.4. For every $\varepsilon>0$ there is a $\delta>0$ such that if $f: M^{n} \rightarrow D \times \mathbf{R}$ is an equivariant map which is a $G-\delta$-equivalence over $D \times[-4,4]$ and a homeomorphism on generalized singular sets, then there is an equivariant $(\pi f)^{-1} \varepsilon$-isotopy, $h_{t}$, of the identity map of $M$, supported on $f^{-1}(D \times[-3,3])$ such that

$$
f^{-1}(D \times(-\infty, 1]) \subset h_{1} f^{-1}(D \times(-\infty, 0)) .
$$

ProOF. Let $\gamma>0$ be given. By induction up the strata of $D^{*}$, applying Corollary 1.7 to isotopies obtained by feathering out (by partitions of unity) piecewise linear slides in the $\mathbf{R}$-coordinate of $D^{*} \times \mathbf{R} \cong M^{*}$, we may assume given an equivariant $(\pi f)^{-1} \gamma$-isotopy $\theta_{t}$ of the identity map of $M$, supported on $f^{-1}(D \times[-3,2])$ such that on some neighborhood, $U$, of $M^{*}$,

$$
\theta_{1}\left(U \cap f^{-1}(D \times[-2, \infty))\right) \subset f^{-1}\left(D \times\left(\frac{3}{2}, \infty\right)\right)
$$


By the proof of the main lemma, which works perfectly well "over $D \times[-4,4]$," there is a $G$-neighborhood, $V$, of $D^{*} \times\left[-\frac{5}{2}, \frac{5}{2}\right]$ and a $\beta>0$ such that if $f$ is a $\beta$-equivalence over $D \times[-4,4]$ and $U$ is as above, there is an equivariant $f^{-1} \gamma$ isotopy, $\lambda_{t}$, of the identity map of $M$, supported on $f^{-1}(D \times[-3,3])$, such that $\lambda_{1}\left(f^{-1} V\right) \subset U$.

If $f$ is a $\delta$-equivalence over $D \times[-4,4]$ and $\mu_{t}$ is any homotopy of $1_{D \times \mathbf{R}}$ supported on $D \times[-4,4]$, then an equivariant $\delta$-lift $\tilde{\mu}_{t}: M \rightarrow M$ will exist and will be isovariant off $f^{-1}$ of the $\delta$-neighborhood of $D^{*} \times \mathbf{R}$. We can use this observation together with our controlled engulfing (cf. $\left[\mathbf{C}_{\mathbf{1}}\right.$, Lemma 3.2]) to produce a $(\pi f)^{-1} \xi$-isotopy, $\tilde{\mu}_{t}$, of the identity map of $M$, supported on $f^{-1}\left(D \times\left[-\frac{3}{2}, 3\right]-V_{1}\right)$, for some neighborhood $V_{1} \subset V$ of $D^{*} \times\left[-\frac{3}{2}, \frac{5}{2}\right]$ such that $\tilde{\mu}_{1} f^{-1}(D \times[-1, \infty)) \cap f^{-1}\left(D \times\left(-\infty, \frac{9}{4}\right]\right)$ is contained in $f^{-1} V$. (Here, $\xi$ depends on $\delta$ via Corollary 2.3.)

Since $\lambda_{t}$ has control over $D \times \mathbf{R}$, a small choice of $\gamma$ will guarantee that

$$
\lambda_{1} f^{-1}\left(D \times\left[\frac{9}{4}, \infty\right)\right)
$$

is in the complement of the support of $\theta_{t}$ and that $\lambda_{1} \tilde{\mu}_{1}\left(f^{-1}(D \times[-1, \infty))\right) \subset$ $f^{-1} D \times[-2, \infty)$. Thus if $\gamma, \xi$ and $\delta<\beta$ are small, successive applications of $\tilde{\mu}_{t}, \lambda_{t}$ and $\theta_{t}$ provide the desired $(\pi f)^{-1} \varepsilon$-isotopy.

6. $\beta$-dominations and $\beta$-maps. Ferry proves the inequivariant versions of the $\beta$-domination theorem and Corollary 2 as a straightforward application of the following two results. We shall state them and discuss the required modifications of Ferry's proofs.

LEMMA 6.1. Let $Y \subset X$ be a locally compact $G$-ANR pair with $Y$ closed in $X$. Then for any open $G$-cover $\alpha$ of $X$ there is an open $G$-cover $\beta$ of $X$ such that if $g: X \rightarrow Z$ is a surjective proper $G-\beta$-map, then there is a proper $\alpha$-domination of pairs $f:(Z, g(Y)) \rightarrow(X, Y)$ with right $\alpha$-inverse $g$.

LEMMA 6.2. Let $\alpha$ be an open $G$-cover of the $G$-manifold $M^{n}$. Then there is an open $G$-cover $\beta$ of $M$ such that if $N^{n}$ is a $G$-manifold and $f:(N, \partial N) \rightarrow$ $(M, \partial M)$ is a proper $G-\beta$-domination of pairs for which $\operatorname{dim} N_{\gamma}^{H}=\operatorname{dim} M_{f(\gamma)}^{H}$ for all fixed-point components, $N_{\gamma}^{H}$, of $N$, then $f$ is a $G$ - $\alpha$-equivalence of pairs.

Ferry proves Lemma 6.1 using the convex structure of separable Hilbert space, $l_{2}$, and the fact that any locally compact (separable metric) ANR embeds as a closed subspace of $l_{2}$. The equivariant analogue is $l_{2}[G]$, the separable Hilbert space whose basis is a free $G$-set. Embeddability of locally compact $G$-ANR's in $l_{2}[G]$ follows from the usual arguments together with the embedding theory in Bredon [Br] (cf. $\left.\left[\mathbf{S W}_{\mathbf{6}}\right]\right)$.

The first requirement for generalizing Ferry's proof of Lemma 6.2 is that any $n$-dimensional $G$-ANR be equivariantly $\beta$-dominated by an $n$-dimensional $G$-CW complex. This follows from the usual nerves-of-covers arguments. The only change necessary is that the application of Ferry's eventual Hurewicz theorem [F, Propositions 3.1-3.3] requires a sequence of neighborhoods for each orbit type in the local representaton $D_{\rho}$, as it will not necessarily be possible to select a single sequence of $G$-connected neighborhoods. Thus, we argue by induction on orbit types and consider only the restriction of the neighborhoods in question to the given fixed-point set. 
7. The equivariant bundle theorem. Let $p: E \rightarrow B$ be a proper equivariant surjection of locally compact, separable metric $G$-spaces. We say that $p$ is equivariantly completely regular if for each $\varepsilon>0$ and each $x \in B$ there is a slice neighborhood $U$ of $x$ such that for each $y \in U$ there is a $G_{y}$-homeomorphism $p^{-1}(y) \rightarrow p^{-1}(x)$ which is $\varepsilon$-close to the inclusion $p^{-1}(y) \subset E$.

We show that a $G$-fibration satisfying the hypothesis of Theorem 2 is equivariantly completely regular, and deduce Theorem 2 from Corollary 1.3 and the following generalization of a theorem of Dyer and Hamstrom [DH], which is based on Michael's selection theorem [M].

PROPOSITION 7.1. Let $p: E \rightarrow B$ be equivariantly completely regular, with $B$ locally finite dimensional, and suppose that the homeomorphism group of each $p^{-1}(x)$ is locally $G_{x}$-contractible. Then $p$ is a $G$-bundle.

PROOF. We wish to find an equivariant local trivialization with the diagonal action over a slice around $x \in B$, so we may as well restrict attention to a slice over which each $p^{-1} y$ is $G_{y}$-homeomorphic to $p^{-1} x$. For simplicity, call this slice $B$, write $G$ for $G_{x}$ and write $F$ for $p^{-1} x$.

Let $P E$ be the subspace of the space of maps from $F$ to $E$ which consists of homeomorphisms from $F$ to fibers of $p$ and let $\pi: P E \rightarrow B$ send $h: F \rightarrow p^{-1} y$ to $y$. Let $G$ act on $P E$ by the diagonal action: $(g \cdot h)(z)=g\left(h\left(g^{-1} z\right)\right)$. Then $\pi$ is equivariant, and if $s: U \rightarrow P E$ is an equivariant section of $\pi$ over a neighborhood $U$ of $x$ in $B$, then there is an equivariant local trivialization (with diagonal action) $\hat{s}: U \times F \rightarrow p^{-1} U$ given by $\hat{s}(y, z)=s(y)(z)$. Conversely, an equivariant local trivialization induces a local section.

Let $P^{\prime} E \subset P E$ be the subspace consisting of those $h: F \rightarrow p^{-1} y$ which are $G_{y}$-equivariant. Then the image of an equivariant local section of $\pi$ always lies in $P^{\prime} E$. In fact, $P^{\prime} E \cap \pi^{-1} y$ is the $G_{y}$-fixed point set of $\pi^{-1} y$, and the restriction $\pi^{\prime}: P^{\prime} E \rightarrow B$ of $\pi$ is isovariant. Thus, the equivariant local sections of $\pi^{\prime}$ are in 1-1 correspondence to the local sections of the orbit map $\pi^{\prime} / G: P^{\prime} E / G \rightarrow B / G$.

We wish to apply Michael's selection theorem [M, Theorem 1.2]. In his language, a selection for the carrier $\phi(y)=\left(\pi^{\prime} / G\right)^{-1}(y)$ is precisely a section of $\pi^{\prime} / G$. Local sections will exist provided that $\phi$ is lower semicontinuous and that the family of fibers of $\pi^{\prime} / G$ is equi- $L C^{n}$ for $n>\operatorname{dim} B$.

The former condition is equivalent to showing that $\pi^{\prime} / G$ is an open map. Since projections to orbit spaces are open, it suffices to show that $\pi^{\prime}$ is open. Let $V$ be an open subspace of $P^{\prime} E$ and let $\left\{y_{i}\right\}$ be a sequence in $B-\pi^{\prime}(V)$ which converges to $y \in B$. By equivariant complete regularity, after possibly passing to a subsequence of $\left\{y_{i}\right\}$, there is a sequence of homeomorphisms $f_{i}: p^{-1} y \rightarrow p^{-1} y_{i}$, such that $f_{i}$ is $G_{y_{i}}$-equivariant, which converges to the identity map of $p^{-1} y$ (recall that $p$ is proper). If $y \in \pi^{\prime}(V)$, there is a $G_{y}$-homeomorphism $f: F \rightarrow p^{-1} y$ in $V$. But then $\left\{f_{i} f\right\}$ converges to $f$ in $P^{\prime} E$.

It now suffices to show that the fibers of $\pi^{\prime}$ are equivariantly equi- $L C^{n}$ in $\mathcal{P}^{\prime} E$, i.e., for each $G_{y}$-neighborhood $U$ of $f \in \pi^{\prime-1} y$ in $P^{\prime} E$ there is a $G_{y}$-neighborhood $V \subset U$ such that any map of a $k$-sphere into $V \cap \pi^{\prime-1}(z), k \leq n$, is nullhomotopic in $U \cap \pi^{\prime-1}(z)$ for any $z \in B$.

Let $\tilde{V}$ be a $G_{y}$-neighborhood of $f$ in $P E$ such that $\tilde{V} \cap P^{\prime} E=V$ and let $V^{\prime}$ be a $G_{y}$-neighborhood of $f$ in $\pi^{-1} y$ such that the $\varepsilon$-neighborhood of $V^{\prime}$ is contained 
in $\tilde{V}$ for some $\varepsilon>0$. Let $U^{\prime}$ be a neighborhood of $f$ in $\pi^{-1} y$ which $G_{y}$-contracts in $V^{\prime}$ and suppose that the $2 \delta$-neighborhood of $f$ in $\pi^{-1} y$ is contained in $U^{\prime}$ for $\delta<\varepsilon$. Take $U$ to be the intersection of the $\delta$-ball around $f$ with the inverse image under $\pi^{\prime}$ of the neighborhood around $y$ specified for $\delta$ in the definition of equivariant complete regularity. Since left translation by $G_{y^{\prime}}$-homeomorphisms is $G_{y^{\prime}}$-equivariant in $P E$, the result follows.

We now show that a $G$-fibration as stated is equivariantly completely regular. This follows from the equivariant $\alpha$-approximation theorem, provided that $p$ satisfies the obvious equivariant analogue of a strongly regular map (cf. [CF]), which holds provided that over a compact neighborhood of each $x \in B$ there is an equivariant lifting function such that for each $\varepsilon>0$ there is a $\delta>0$ such that equivariant $\delta$-homotopies into $B$ lift to equivariant $\varepsilon$-homotopies into $E$. Since $p$ is proper, this will hold by a Lebesgue number argument for any equivariant regular lifting function. Since $B$ is metric, such a lifting function exists by the usual arguments (cf. $\left.\left[\mathbf{S W}_{\mathbf{1}}\right]\right)$.

REMARKS. In fact, Michael's argument has an obvious generalization to actions by finite groups, which would provide equivariant sections of $\pi$ directly.

8. The equivariant thin $h$-cobordism theorem. Let $(W, M)$ be a $G-\beta h$ cobordism with controlling deformation retraction $r: W \rightarrow M$. Let $\bar{W}$ be the union of $W$ with an external collar on $M$ and let $\bar{r}: \bar{W} \rightarrow M \times I$ be $r$ on $W$ and the identity map on the collar. Since $r$ is close to the projection map on the low dimensional strata, we may assume, with slight loss of control, that $\bar{r}$ is a homeomorphism there. Thus, our thin $h$-cobordism will follow from the $\alpha$-approximation theorem provided that $\bar{r}$ is a well enough controlled homotopy equivalence on the boundary.

It is enough to show that for $\beta$ small, the inclusion $\overline{\partial W-M} \subset W$ is a $G-r^{-1} \gamma$ equivalence for $\gamma$ small. First, replace $\overline{\partial W-M}$ with $M_{1}$, the complement in $\partial W$ of the union of $M$ with a small (with respect to $\beta$ ) open collar on $\partial M$.

We shall apply the locally compact version of Quinn's eventual Hurewicz theorem. As before, since Quinn's argument is an induction on skeleta, it suffices to show that Quinn's hypotheses hold simultaneously in each fixed-point set. For this we note that since $W$ is locally linear and local representations are constant along isovariant arcs, a quick induction shows that each fixed-point component of $W$ is an equivariant $\beta$ - $h$-cobordism between the pertinent components of $M$ and $M_{1}$.

Consider the end $r: W-M \rightarrow M$ and let $U_{0} \supset \cdots \supset U_{2^{n+3}}$ be complements of small collar neighborhoods of $M_{1}$ in $W$. Then for $\beta^{\prime}$ slightly larger than $\beta$ there is a $G$ - $\beta^{\prime}$-deformation retraction of each $U_{i}$ onto $M$. Moreover, each inclusion $U_{j}-U_{j+1} \subset U_{j-1}-U_{j+2}$ is equivariantly $\left(\beta^{\prime}, 1\right)$-connected because the identity map of $\overline{\partial W-M}$ is $(\beta, 1)$-connected. Thus, as in $\left[\mathbf{Q}_{\mathbf{1}}, 5.3\right]$, there is a small equivariant deformation of $W-M \operatorname{rel} M_{1}$ into a small collar around $M_{1}$.

\section{REFERENCES}

[Bi] R. H. Bing, Radial engulfing, Conf. on the Topology of Manifolds (M.S.U., 1967), Prindle, Weber and Schmidt, Boston, Mass, 1968, pp. 1-18.

[Br] G. Bredon, Introduction to compact transformation groups, Academic Press, New York, 1972, 
[BQ] W. Browder and F. Quinn, A surgery theory for $G$-manifolds and stratified sets, Manifolds, Tokyo 1973 (Proc. Internat. Conf., Tokyo, 1973), Univ. Tokyo Press, Tokyo, 1975, pp. 27-36.

[Bry] J. L. Bryant, Approximating embeddings of polyhedra in codimension three, Trans. Amer. Math. Soc. 170 (1972), 85-95.

[BS] J. L. Bryant and C. L. Seebeck III, Locally nice embeddings in codimension three, Quart. J. Math. Oxford 21 (1970), 265-272.

[C $\mathbf{C}_{1}$ T. A. Chapman, Approximation results in topological manifolds, Mem. Amer. Math. Soc. No. 251, 1981.

$\left[\mathbf{C}_{\mathbf{2}}\right] \ldots$, Controlled simple homotopy theory, Lecture Notes in Math., vol. 1009, Springer-Verlag, 1983.

[C] _ Lectures on Hilbert cube manifolds, CBMS Regional Conf. Ser. in Math., no. 28, Amer. Math. Soc., Providence, R.I., 1976.

[CF] T. A. Chapman and S. Ferry, Approximating homotopy equivalences by homeomorphisms, Amer. J. Math. 101 (1979), 583-607.

[DH] E. Dyer and M.-E. Hamstrom, Completely regular mappings, Fund. Math. 45 (1957), 103-118.

[E] R. D. Edwards, Approximating certain cell-like maps by homeomorphisms, manuscript.

[EK] R. D. Edwards and R. C. Kirby, Deformations of spaces of imbeddings, Ann. of Math. (2) 93 (1971), 63-88.

[F] S. Ferry, Homotoping $\varepsilon$-maps to homeomorphisms, Amer. J. Math. 101 (1979), 567-582.

[Hav] W. Haver, Mappings between ANRs that are fine homotopy equivalences, Pacific J. Math. 58 (1975), 457-461.

[H] J. F. P. Hudson, Piecewise linear topology, Benjamin, New York, 1969.

[II $\left.{ }_{1}\right]$ S. Illman, Whitehead torsion and group actions, Ann. Acad. Sci. Fenn. Ser. A 588 (1974), 1-44.

$\left[\mathrm{Il}_{2}\right]$ - Recognition of linear actions on spheres, Trans. Amer. Math. Soc. 274 (1982), 445-478.

$\left[\mathrm{Il}_{\mathbf{3}}\right] \ldots$, Approximation of $G$-maps by maps in general position and imbeddings of $G$-complexes, Trans. Amer. Math. Soc. 262 (1980), 113-157.

[J] J. W. Jaworowski, Extensions of $G$-maps and Euclidean G-retracts, Math. Z. 146 (1976), 143-148.

[KS] R. C. Kirby and L. C. Siebenmann, Foundational essays on topological manifolds, smoothings and triangulations, Ann. of Math. Studies, no. 88, Princeton Univ. Press, Princeton, N.J., 1977.

[L] R. K. Lashof, The equivariant extension theorem, Proc. Amer. Math. Soc. 83 (1981), 138-140.

[LR] R. Lashof and M. Rothenberg, G-smoothing theory, Proc. Sympos. Pure Math., vol. 32, Amer. Math. Soc., Providence, R.I., 1978, pp. 221-266.

[M] E. Michael, Continuous selections. II, Ann. of Math. (2) 64 (1956), 562-580.

[Mil] R. T. Miller, Approximating codimension 3 embeddings, Ann. of Math. (2) 95 (1972), 406-416.

[Q1] F. Quinn, Ends of maps. I, Ann. of Math. (2) 110 (1979), 275-331.

[Q2] , Ends of maps. II, Invent. Math. 68 (1982), 353-424.

[R] M. Rothenberg, Torsion invariants and finite transformation groups, Proc. Sympos. Pure Math., vol. 32, Amer. Math. Soc., Providence, R.I., 1978, pp. 267-311.

[Ru] T. B. Rushing, Topological embeddings, Academic Press, New York, 1973.

[S] M. Steinberger, The equivariant s-cobordism theorem, preprint.

[Si $\left.{ }_{1}\right]$ L. C. Siebenmann, Approximating cellular maps by homeomorphisms, Topology 11 (1972), 271294.

[Si $\left.\mathbf{S}_{2}\right]$, Deformation of homeomorphisms on stratified sets, Comment. Math. Helv. 47 (1972), 123163.

[SGH] L. C. Siebenmann, L. Guillou, and H. Hähl, Les voisinages ouverts réguliers: critères homotopiques d'existence, Ann. Sci. Ecole Norm. Sup. 7 (1974), 431-462.

[Sp] E. H. Spanier, Algebraic topology, McGraw-Hill, New York, 1966.

[SW $\mathbf{S W}_{1}$ M. Steinberger and J. E. West, Covering homotopy properties of maps between $C W$ complexes or $A N R$ 's, Proc. Amer. Math. Soc. 92 (1984), 573-577.

[SW $\mathbf{S}]$, Equivariant $h$-cobordisms and finiteness obstructions, Bull. Amer. Math. Soc. (N.S.) 12 (1985), 217-270.

[SW $\mathbf{S}$ ] Topology, Stefan Banach Center, Warsaw (to appear).

[SW $\mathbf{S W}_{\mathbf{4}}$, Equivariant handles in finite group actions, Proc. 1985 Univ. of Georgia Topology Conf. (to appear).

$\left[\mathbf{S W}_{\mathbf{5}}\right]$ - Controlled finiteness is the obstruction to equivariant handle decomposition, preprint.

[SW $\left.\mathbf{S W}_{\mathbf{6}}\right]$, Equivariant ANR fibrations and Hilbert cube manifold bundles (in preparation). 
[SW $\left.\mathbf{S W}_{\mathbf{7}}\right]$, Equivariant controlled simple homotopy theory (in preparation).

[SW $\mathbf{S}]$, Approximation by equivariant homeomorphisms. II (in preparation).

Department OF MATHEMATICAL SCIENCES, NORThern Illinois UNiversity, DE KALB, ILLINOIS 60115

Department of Mathematics, Cornell University, Ithaca, New York 14853 (Current address of J. West)

Current address (M. Steinberger): Department of Mathematics and Computer Science, Rutgers University, Newark, New Jersey 07102 